\title{
CIANOBACTERIAS Y RIESGOS POTENCIALES DE TOXICIDAD EN AGUAS CONTINENTALES DE CHILE
}

(Cyanobacteria and potential risks of toxicity in continental waters of Chile)

\author{
Victoriano Campos*, Sandra Lisperguer*, Jurgen Weckesser**, \\ Alejandra Vera*, Daniela Muñoz* \\ * Laboratorio de Microbiologia Ambiental, Instituto de Biología, \\ Facultad de Ciencias Básicas y Matemáticas, Pontificia Universidad \\ Católica de Valparaíso, Casilla 4059, Valparaíso, Chile \\ ** Universitat Freiburg, Institut fúr Biologie II, Mikrobiologie, \\ Schánzlestraße 1, D-79104 Freiburg i.Br, Germany
}

Palabras clave: Cianobacterias, toxicidad, aguas continentales, Chile

Key words: Cyanobacteria, toxicity, continental water, Chile

\section{RESUMEN}

Las cianobacterias son microorganismos fototróficos oxigénicos, con una amplia distribución, ya que han sido reportados en aguas dulces o saladas de los cinco continentes. Eventualmente pueden desarrollarse en grandes masas, denominadas «blooms» (florecimientos), generados, usualmente, por efectos antropogénicos. Varias especies de cianobacterias pueden producir potentes neurotoxinas y hepatotoxinas, las cuales presentan serias dificultades para ser eliminadas del agua. La intoxicación con algunas de estas toxinas puede ser grave causando la muerte de animales o el hombre y se sospecha que la ingestión aún de microdosis, pueda inducir la formación de tumores. La distribución de cianobacterias de aguas continentales de Chile, se presenta desde el extremo Norte hasta el territorio Antártico. Se ha determinado en diferentes regiones la presencia de especies tóxicas y la formación de florecimientos. Mediante cromatografía líquida de alta presión (HPLC) y espectrometría de masas (MALDITOF) (PSD), se ha determinado en diferentes cuerpos de agua la cianotoxina microcistina. Finalmente se sugieren recomendaciones para tomar conocimiento y establecer controles que eviten el riesgo a su exposición.

\section{INTRODUCCION}

Por muchos años las cianobacterias fueron consideradas algas verde azuladas debido a su fototrofía oxigénica. Stanier et al. en 1978, propusieron la inclusión de este grupo en el Código Internacional de Nomenclatura

\section{ABSTRACT}

Cyanobacteria are phototropic and oxygenic microorganisms that are widely distributed and reported to be present in freshwater or seawater from the five continents. They can eventually develop into large masses called «blooms» which are usually generated by anthropogenic effects. Several species of cyanobacteria can produce potent neurotoxins and hepatotoxins, which are difficult to eliminate from water. The intoxication with some of these toxins can become severe causing death in animals and man and it is suspected that ingestion of even microdoses of them can induce tumor appearance. The distribution of cyanobacteria in continental waters of Chile has been recorded from uppermost north of Chile up to the Antarctic territory. The presence of toxic species as well as the development of blooms have been detected in different regions.The mycrocystin cyanotoxin has been assessed in different masses of water by means of high pressure liquid chromatography (HPLC) together with mass spectrometry (MALDI-TOF) (PSD). Finally it is recommended to make analysis in order to establish controls that avoid risk of exposure to these cyanotoxins.

\section{Bacteriana.}

La estructura fina de las cianobacterias es bien conocida. Son organismos fotoautótrofos, que realizan la fotosíntesis con liberación de oxígeno, presentan clorofila a como pigmento fotosintético primario y ficobiliproteínas como pigmentos auxiliares (Bryantt, 1986). 
Entre las ficobilinas se encuentran las ficocianinas azules con máximo de absorción a 625 nm que junto con la clorofila a verde, dan a estas bacterias el característico color verde-azulado. Algunas cianobacterias producen ficoeritrina de color rojo con un máximo de absorción a $550 \mathrm{~nm}$, y que les otorga un color rojo o marrón.

Algunas especies son capaces de cambiar a una fotosíntesis anoxigénica, lo que ocurre en ambientes con una alta concentración de $\mathrm{H}_{2} \mathrm{~S}$ (3mM) (Cohen et al., 1986).

Además, son capaces de adaptarse a diferentes condiciones de luz por su habilidad de cambiar su aparato fotosintético modificando el área de la superficie fotosintética de los tilacoides ( Golecki \& Drews, 1982) o cambiando el tamaño y composición de las ficobilinas (Cohen-Bazire \& Bryant, 1982 ). Adicionalmente, las cianobacterias pueden protegerse de la intensidad de luz de alta energía, por medio de pigmentos como carotenoides y xantófilas y posiblemente con otras estructuras como las vacuolas de gas o las vainas (Van Liere \& Walsby, 1982). Así mismo, algunas cianobacterias tienen la capacidad de moverse en un campo de luz por medio de vacuolas de gas que regulan su posición en una columna de agua. En muchas cianobacterias filamentosas su movilidad por deslizamiento acompañada de fototaxis, les permite moverse hacia las áreas de óptimas condiciones de luz (Castenholz, 1982). Así, en tapetes microbianos las cianobacterias filamentosas pueden migrar a diferentes niveles en respuesta a la intensidad de la luz.

Las cianobacterias tienen una serie de propiedades que les permite desarrollarse con éxito y dominar una amplia variedad de ambientes iluminados, por lo cual su principal factor limitante sería la luz.

Las Cianobacterias predominantemente son fotoautotróficas, sólo algunas son capaces de heterotrofía en condiciones de laboratorio. La habilidad para crecer fotoautotróficamente utilizando compuestos orgánicos como única fuente de carbono y luz como fuente de energía es relativamente usual en cianobacterias cultivadas, mientras la habilidad de crecer en oscuridad utilizando compuestos orgánicos como única fuente de carbono y energía es muy restringida (Rippka et al., 1979).

La habilidad de muchas cianobacterias de fijar nitrógeno atmosférico les permite desarrollarse en hábitats con poca disponibilidad de nitrógeno combinado. Sin embargo, las cianobacterias viven en ambientes con oxígeno y lo producen durante la fotosíntesis. La nitrogenasa que es la enzima responsable de la fijación de $\mathrm{N}_{2}$ es sensible al oxígeno o reprimida por él. Así, algunas bacterias filamentosas han resuelto este problema separando espacial y temporalmente el proceso de fotosíntesis oxigénica de la fijación de dinitrógeno generando células altamente diferenciadas conocidas como heterocistos (Bothe, 1982).

Las cianobacterias se pueden encontrar en ambientes con diferentes rangos de temperatura, muchas son mesófilas con crecimiento óptimo entre $20^{\circ}$ y $35^{\circ} \mathrm{C}$. Las cianobacterias oceánicas donde la temperatura es más moderada, presentan una máxima cercana a $30^{\circ} \mathrm{C}$ (Waterbury et al., 1986). En ambientes extremos como la
Antártica, se encuentran especies psicrófilas como Chrococcidiopsis, cuya temperatura máxima de crecimiento es de $20^{\circ} \mathrm{C}$ (Seaburg, 1981).

Muchas cianobacterias presentan temperaturas óptimas de crecimiento más altas que la de muchas algas eucariotas, llegando algunas a tolerar hasta $74^{\circ} \mathrm{C}$ en manantiales calientes (Castenholz, 1984).

También, se pueden encontrar en el interior de rocas, denominadas endolíticas, en áreas secas y frías, en tapetes, en lagos y en el plancton o en procesos de simbiosis con eucariotas, debido a su capacidad de fijación de nitrógeno, como es el caso de los cianolíquenes, muy comúnes en diversas regiones del mundo.

Por otra parte, pueden desarrollarse en ambientes con diferente grado de salinidad. Los hábitats de agua dulce contienen diversidad y frecuentemente altas poblaciones de cianobacterias. En el ambiente marino suelen encontrarse dos tipos: halotolerantes y halófilas. Dominan muchos ambientes hipersalinos como lagunas y lagos (Bauld, 1981; Javor \& Castenholz, 1981; Stal, 1995). A pesar de su frecuencia en ambientes hipersalinos, sólo un grupo restringido es halófilo (Brock, 1976).

Referente al pH, las cianobacterias requieren un $\mathrm{pH}$ neutro o levemente alcalino, habitualmente no se las encuentra en ambientes con un $\mathrm{pH}$ muy ácido. En sus aislamientos de aguas termales de $\mathrm{pH}$ ácido, crecen normalmente a $\mathrm{pH}$ neutro, lo que indicaría más bien que son ácidotolerantes que acidófilas (Rippka et al., 1981). Muy pocas especies pueden desarrollarse en un ambiente altamente alcalino, como Spirulina platenses, dominante en lagos alcalinos con pH óptimo entre 8-11 (Ciferri, 1983).

Frecuentemente, las cianobacterias pueden encontrarse en condiciones de sequedad extrema tanto en las zonas tropicales como en la Antártica (Fogg et al., 1973). Aunque, se pueden encontrar en condiciones extremas de temperatura, salinidad, pH o sequedad, en el laboratorio presentan óptimos crecimientos a condiciones más moderadas, por lo que parecen ser tolerantes a condiciones extremas más que extremófilos obligados.

Por la capacidad de las cianobacterias de poder desarrollarse en variados ambientes de condiciones extremas y su independencia metabólica como fotoautotrofos, Fridmann (2001), microbiólogo de la NASA ha propuesto a la cianobacteria Chroococcidiopsis como microorganismo para iniciar la colonización de Marte.

\section{Florecimientos y toxicidad}

El desarrollo de florecimientos de cianobacterias es un fenómeno muy conocido en especial en cuerpos de agua eutróficos, ya que se han reportado alrededor de todo el mundo, en Europa, Australia, América y Africa del Sur (Carmichael, 1994; Azevedo et al., 1994; Scarafia et al., 1995).

Bajo determinadas condiciones ambientales de temperatura y de nutrientes particularmente fósforo y nitrógeno, en épocas de primavera o verano las ciano- 
bacterias pueden desarrollarse en grandes masas denominadas florecimientos (blooms), presentando una estratificación en la columna de agua (Paerl, 1996), la creciente eutrofización de los ambientes acuáticos favorece el desarrollo de los florecimientos y más del 50\% de éstos, presentan toxicidad (Sevomen \& James, 1999), pudiendo contener potentes toxinas, siendo las más comunes las neurotoxinas y las hepatotoxinas (Codd, 1995).

Al parecer, el fósforo jugaría un papel importante tanto en el desarrollo de la población de cianobacterias, como de las toxinas, como se ha demostrado en trabajos realizados en Anabaena con hepatotoxinas (Rapala et al., 1997). Así mismo, se ha establecido que altos niveles de nitrógeno y fósforo favorecen el crecimiento de cepas tóxicas de Microcystis sobre las no tóxicas (Vezie et al., 2002).

Uno de los grupos de cianotoxinas, más estudiados corresponde a las neurotoxinas, que pueden actuar bloqueando la despolarización post-sináptica como la Anatoxina-a, o bloqueando la acetilcolinesterasa como la Anatoxina a-S, o bloqueando los canales de sodio como la saxitoxina y neosaxitoxina. Las neurotoxinas son producidas por géneros como: Anabaena, Oscillatoria, Microcystis, Aphanizomenon.

Otras cianotoxinas que se presentan con más regularidad son las hepatotoxinas que corresponden a péptidos cíclicos que bloquean las proteínas fosfatasas (Eriksson et al., 1990; Yoshizarva et al., 1990) y producen hemorragia en el hígado, también se ha podido revelar por diferentes autores que las toxinas desarticulan el citoesqueleto del hepatocito. Diversas especies o cepas de cianobacterias pueden producir hepatotoxinas, las que pueden estar constituídas por pentapéptidos como nodularina, aislada de Nodularia o constituídas por heptapéptidos, como las microcistinas, inicialmente aislada de Microcystis y posteriormente de Anabaena, Nostoc y Oscillatoria (Tabla 1). El mecanismo de acción de las toxinas hepatotóxicas y neurotóxicas se describe en Carmichael (1994).

Diversos trabajos indican la muerte de animales por cianotoxinas en diferentes lugares del mundo (Skulberg et al., 1994; Codd et al., 1989; Carmichael \& Falconer, 1993) y también problemas de salud humana, donde la posibilidad que toxinas en bajas concentraciones sean potentes promotores de cáncer al hígado, lo que hace suponer que sus altas tasas son debidas al repetido consumo de microdosis de toxinas en el agua potable (Carmichael, 1994).

Así mismo, pacientes con tratamiento de hemodiálisis murieron al usar agua contaminada con microcistina de un reservorio brasileño (Jochinsen et al., 1998; Pouria et al., 1998).

Por otra parte, las cianobacterias también producen péptidos no tóxicos, muchas veces simultáneamente en el mismo florecimiento (Neumann et al., 2000); muchos de ellos como la cianopeptolina muestran importantes actividades biológicas. Una revisión de la estructura y actividad biológica de péptidos no tóxicos se encuentra en Weckesser et al. (1996).

\section{Cianobacterias en Aguas Continentales de Chile}

El territorio continental americano de Chile se extiende por más de $4270 \mathrm{Km}$ con un ancho máximo de 435 Km y un mínimo de $90 \mathrm{Km}$ entre la Cordillera de los Andes y el Mar Chileno del Océano Pacífico. En esta vasta extensión existe una gran variedad climática, en general una zona de clima desértico en el norte, de clima templado mediterráneo en el centro y un clima lluvioso y frío del sur, sin considerar la situación climática del territorio Antártico chileno y las múltiples variaciones climáticas expresadas en microclimas particulares en muchas áreas del territorio continental.

Así, para fines prácticos, hemos dividido el territorio continental americano chileno en tres zonas o áreas, una zona Norte con un ambiente desértico, una zona Centro con clima mediterráneo, que tiene además el mayor consumo de agua debido a la concentración de la población y una zona Sur lluviosa donde se encuentra la mayor concentración de los grandes Lagos de Chile. No se considera el territorio Antártico chileno, donde indudablemente se dispone de información científica de la presencia de diferentes cianobacterias, pero con una escasa posibilidad de significar riesgo de toxicidad.

En el presente trabajo analizamos la presencia de cianobacterias en el Norte de Chile, II región de Antofagasta, en las regiones V, VI y Metropolitana en el centro y en las regiones VII, IX y X de la zona Sur.

En el norte, específicamente en un ambiente extremo como es el Salar de Atacama, las cianobacterias se presentan en lagunas hipersalinas, tapetes microbianos, florescencias salinas y también cuerpos de agua con baja concentración de sal. Si bien las cianobacterias no son organismos extremófilos, pueden ser tolerantes a condiciones extremas y desarrollarse significativamente en estos ambientes, que se caracterizan por escasa pluviometría, como por ejemplo la ciudad de Arica, que registra menos de $1 \mathrm{~mm}$ como promedio anual. Sin embargo, la zona norte presenta altas cumbres en la Cordillera de Los Andes (Altiplano) con más de 5.000 m, que tienen lluvias y nieves provenientes del sistema amazónico que aportan agua al desierto en cursos de agua que arrastran altos contenidos salinos dando origen a ríos y lagunas hipersalinas.

Así, tanto en aguas de bajo contenido de salinidad como en lagunas saladas como Tebenquiche (Fig. 1-4) y Chaxa (Fig. 1-5) se ha logrado determinar la presencia de cianobacterias como: Anabaena,(Fig. 2-9) Gleocapsa, Gloethece, Nostoc, Oscillatoria y Synechococcus. (Zúñiga et al., 1991; Campos et al., 1990) (Tabla 2).

En los tapetes microbianos extraídos en las zonas de las lagunas (Fig. 2- 8), la cianobacteria dominante resultó ser Oscillatoria (Fig. 2-7), posiblemente por su ventaja de cambiar de posición en el tapete de acuerdo a la intensidad de la luminosidad 

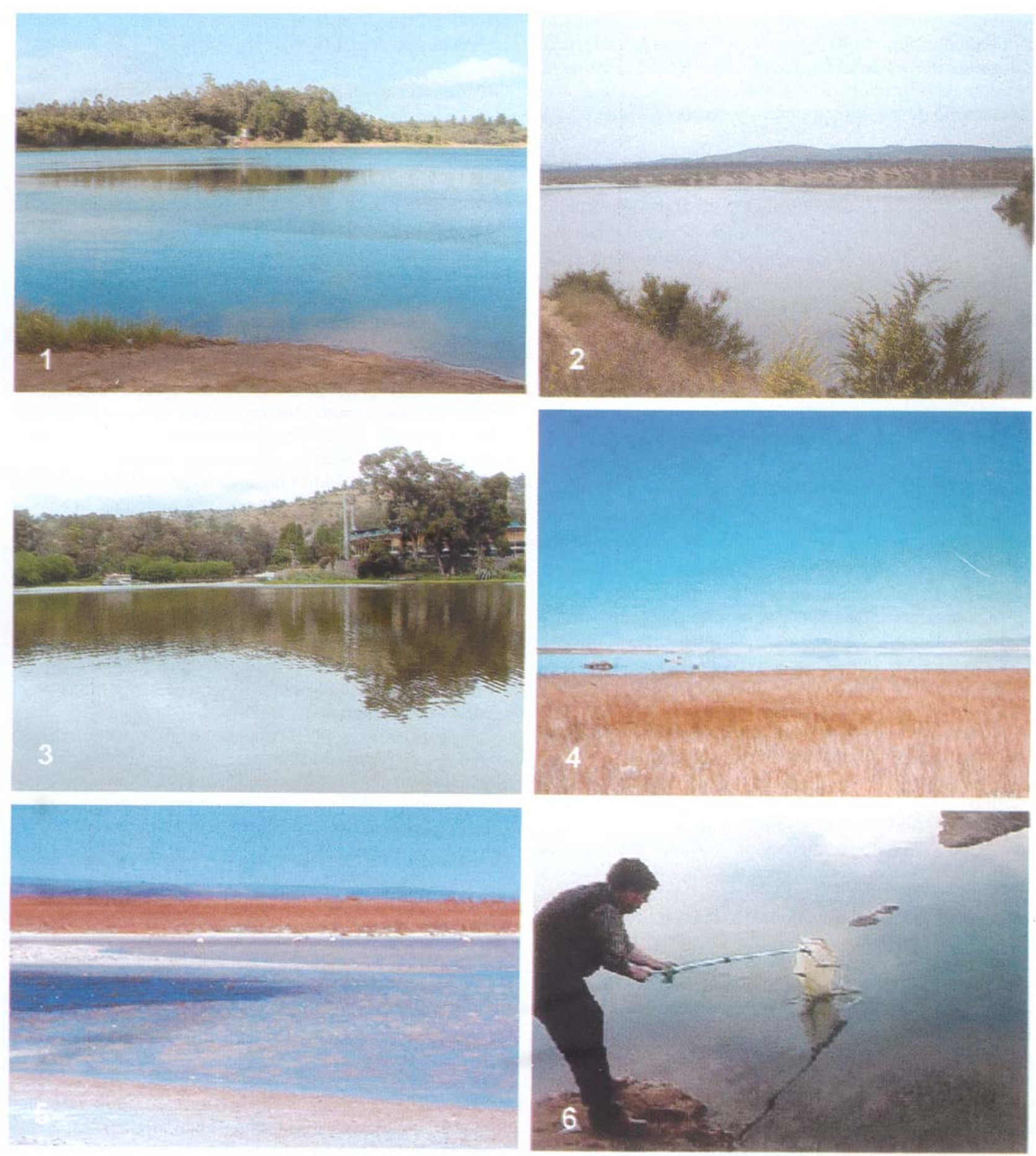

Figura 1.- 1. Lago Peñuelas (V Región), 2. Embalse Los Aromos (V Región), 3. Laguna Sausalito (V Región), 4. Laguna hipersalina de Tebenquiche (II Región), 5. Laguna hipersalina de Chaxa (II Región), 6. Toma de muestra en Lago Peñuelas. 

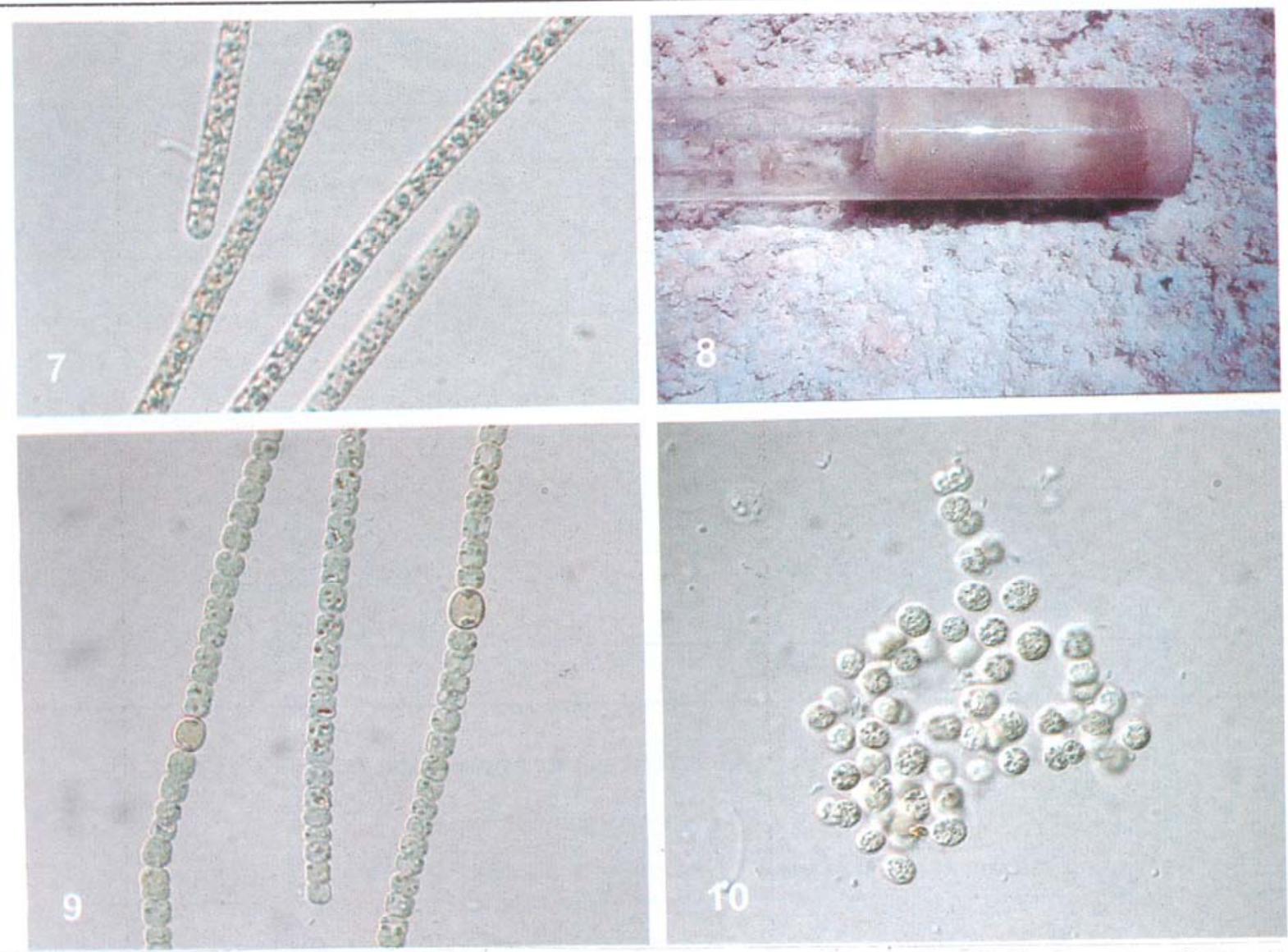

Figura 2.- 7. Oscillatoria sp. 1000x, 8. Tapete microbiano de Laguna hipersalina, 9. Anabaena sp. $1000 x, 10$ Microcystis sp. 1000x.

También se pueden encontrar cianobacterias en un fenómeno de supervivencia que hemos denominado "bubble life», que se produce al evaporarse el agua y perder permeabilidad el suelo, los gases provenientes de los sedimentos generan burbujas que se consolidan en diferentes formas conteniendo en su interior diversos microorganismos entre ellos cianobacterias, que pueden mantenerse latentes por un largo período de tiempo. Las cianobacterias en la zona desértica, además de presentarse en diferentes tipos de agua, ocupan nichos muy sofisticados como rocas salinas, tapetes microbianos y «bubble life».

La zona central del país tiene especial importancia en relación a toxicidad del agua por cianotoxinas, reúne la más alta concentración de la población, más de 7.000.000 de habitantes, por lo cual los requerimientos de agua de bebida son los más altos del pais, el régimen de lluvias es estacional no superando los $600 \mathrm{ml} / \mathrm{año}$, lo que ha demandado la construcción de embalses. Por lo tanto, el estudio se ha centrado especialmente en las regiones V, VI y Metropolitana. Comprendiendo en la V Región el Lago Peñuelas (Fig. 1-1), Laguna La Luz de Curauma, Laguna Sausalito (Fig. 1-3), Embalse Los Aromos (Fig. 1-2) y los Esteros Marga-Marga y Reñaca. De la región Metropolitana existe información de la Laguna Aculeo y de la VI Región del Embalse de Rapel (Cabrera et al., 1977; Vila et al., 1987; Muhlhauser, 1990).Fundamentalmente los géneros determinados fueron: Anabaena, Microcystis (Fig. 210), Oscillatoria y Spirullina, habiéndose registrado florecimientos de Anabaena y Microcystis (Tabla 3).

En la zona Sur, se consideran los trabajos realizados entre las ciudades de Concepción y Puerto Mont, comprendiendo las Regiones del Bio-Bio, la Araucanía y de Los Lagos.

Si bien muchos de los estudios tienen un especial interés limnológico en variaciones estacionales y productividad primaria en general, consignan las especies de zooplancton y fitoplancton incluyendo especies de cianobacterias. En la Tabla 4, se presentan los datos referentes a la Región del Bío-Bío que incluye: Las Lagunas, Chica de San Pedro, Lo Mendez, Tres Pascualas, La Posada y La Marisma de Rocuant ( Dellarosa et al., 1976; Parra et al., 1976; Campos et al., 1999).

En la Tabla 5 se consigna la información de la Región de la Araucanía y de la Región de Los Lagos (Campos et al., 1983; 1987 a, b, 1988, 1990, 1992 a, b).

\section{Riesgos Potenciales de Toxicidad}

Se ha determinado la presencia de diferentes géneros de cianobacterias en todo el territorio continental 
Tabla 1: Cianotoxinas de mayor importancia

\begin{tabular}{|c|c|c|}
\hline & & $\begin{array}{l}\text { Producidas por especies o cepas de los } \\
\text { Géneros }\end{array}$ \\
\hline $\begin{array}{l}\text { Neurotoxinas } \\
\text { Eloqueo de la despolarización post- } \\
\text { sináptica }\end{array}$ & Anatoxina-a & $\begin{array}{l}\text { Arrabaena } \\
\text { Oscillabria } \\
\text { Aphanizom enon }\end{array}$ \\
\hline Bloqueo de acetilcolinesterasa & Anatoxina-a(s) & Arrabaena \\
\hline Bloqueo de canales de sodio & Saxitoxinas & $\begin{array}{l}\text { Arabaena } \\
\text { Aphanizom enon } \\
\text { Lyngbya }\end{array}$ \\
\hline \multicolumn{3}{|l|}{ Hepatotoxinas } \\
\hline $\begin{array}{l}\text { Bloqueo de proteínas fosfatasas } \\
\text { Atteración de microtúbulos y } \\
\text { micrólamentos del citoesqueleto del } \\
\text { hepatocito }\end{array}$ & Microcistinas & $\begin{array}{l}\text { Morocystis } \\
\text { Arabaena } \\
\text { hostoc } \\
\text { Oscillatoria } \\
\text { Planitotrix }\end{array}$ \\
\hline & Nodularina & noodularia \\
\hline
\end{tabular}

Tabla 2: Géneros de Cianobacterias en la II Región de Antofagasta, Salar de Atacama

\begin{tabular}{|c|c|c|c|c|c|c|}
\hline \multirow{2}{*}{$\begin{array}{c}\text { Cuerpos de } \\
\text { Agua }\end{array}$} & \multicolumn{6}{|c|}{ Géneros de Cianobacteras } \\
\hline & Anabaena & Gloeocapsa & Gloeothece & Nostoc & Oscillatoria & Synechococcus \\
\hline $\begin{array}{l}\text { Laguna } \\
\text { Tebenquiche }\end{array}$ & $\mathrm{x}$ & & & $\mathrm{x}$ & $\mathrm{x}$ & \\
\hline $\begin{array}{l}\text { Laguna } \\
\text { Chaxa }\end{array}$ & $\mathrm{x}$ & & & $\mathrm{x}$ & $\mathrm{x}$ & \\
\hline Tilopozo & & $\mathrm{X}$ & $\mathrm{x}$ & & $\mathrm{x}$ & $\mathrm{x}$ \\
\hline
\end{tabular}

Tabla 3: Géneros de Cianobacterias en la V Región de Valparaíso, VI Región del Libertador Bernardo O'Higgins y Región Metropolitana

\begin{tabular}{|c|c|c|c|c|}
\hline \multirow{6}{*}{$\begin{array}{l}\text { CUERPOSDE A GUA } \\
\text { Lago Peñuelas, V Región } \\
\text { Laguna h Lux de Curauma, V } \\
\text { Región } \\
\text { Laguna Sausalito, V Región }\end{array}$} & \multicolumn{4}{|c|}{ Género de Cianobacterias } \\
\hline & Anabaena & Microcystis & Oscillatoria & Spirullina \\
\hline & $+\mathrm{f}^{*}$ & & + & \\
\hline & & & + & + \\
\hline & & & & \\
\hline & + & & + & + \\
\hline Emb ake Los Aromos, V Regín & + & + & & \\
\hline Es tero Marga-Maga, V Región & & & + & \\
\hline $\begin{array}{l}\text { Laguna Aculeo, Región } \\
\text { Metrop olitana }\end{array}$ & & $+\mathrm{f}^{*}$ & & \\
\hline Emb ake Rapel, VI región & + & + & + & \\
\hline
\end{tabular}

*: se utilizan los nombres en uso que pueden no estar de acuerdo a la nomenclatura geográfica 
Tabla 4: Géneros de Cianobacterias en la VIII Región del Bío-Bío

\begin{tabular}{|l|c|c|c|c|}
\hline \multirow{2}{*}{ Cuerpos de Agua } & \multicolumn{4}{c|}{ Género de Cianob acterias } \\
\cline { 2 - 5 } & Anabaena & Merismopedia & Microcystis & Oscillatoria \\
\hline Laguna Chica San Pedro & & + & $+\mathrm{f}^{*}$ & + \\
\hline Laguna Posada & & & $+\mathrm{f}^{*}$ & \\
\hline Marisma de Roc uant & + & + & $+\mathrm{f}^{*}$ & + \\
\hline Laguna Lo Mendex & & + & $+\mathrm{f}^{*}$ & \\
\hline Laguna tres Pascuahs & & & $+\mathrm{f}^{*}$ & \\
\hline
\end{tabular}

$+f^{*}$ : donde se han señalado florecimientos (blooms)

Tabla 5: Géneros de Cianobacterias en la IX Región de la Araucanía y X Región de Los Lagos

\begin{tabular}{|l|c|c|c|c|c|c|c|c|}
\hline \multirow{2}{*}{$\begin{array}{c}\text { Cuerpos de } \\
\text { Agua }\end{array}$} & \multicolumn{7}{|c|}{ Géneros de Cianobacterias } \\
\cline { 2 - 10 } & $\begin{array}{c}\text { Ana- } \\
\text { baena }\end{array}$ & $\begin{array}{c}\text { Apha- } \\
\text { nothece }\end{array}$ & $\begin{array}{c}\text { Chroo- } \\
\text { coccus }\end{array}$ & $\begin{array}{c}\text { Gomphos - } \\
\text { phaeria }\end{array}$ & $\begin{array}{c}\text { Merismo- } \\
\text { pedia }\end{array}$ & $\begin{array}{c}\text { Micto- } \\
\text { cystis }\end{array}$ & $\begin{array}{c}\text { Sscilla- } \\
\text { toria }\end{array}$ & $\begin{array}{c}\text { Spiru- } \\
\text { llina }\end{array}$ \\
\hline Caburga & & & + & + & & & & \\
\hline Villarica & + & & & + & & + & + & \\
\hline Riñihue & + & & + & & & & + & \\
\hline Ranco & + & + & + & + & & + & & \\
\hline Puyehue & + & & & + & + & + & & \\
\hline Rupanco & + & & + & & & + & & \\
\hline $\begin{array}{l}\text { Todos Los } \\
\text { Santos }\end{array}$ & + & & + & & & & & + \\
\hline Llanquihue & & + & + & + & & + & + & \\
\hline
\end{tabular}

de Chile. Adicionalmente, se debe considerar la presencia de géneros de cianobacterias potencialmente tóxicas como son: Anabaena, Oscillatoria y Microcystis . Por otra parte se han determinado florecimientos en especial de Microcystis y de Anabaena y en el de Microcystis se determinó la toxina hepatotóxica microcistina.

Los mayores riesgos estarían vinculados a zonas de alta densidad de población, donde el agua de abastecimiento para agua potable proviene de lagos, lagunas o embalses. Usualmente las ciudades cercanas a la cordillera de los Andes obtienen el agua de cursos de agua o punteras en alta montaña sin posibilidad de crecimientos significativos de cianobacterias. El mayor riesgo lo tendrían las ciudades costeras abastecidas por embalses.

Las Instituciones responsables deberían controlar el incremento de la eutrofización de los lagos por efecto de: la actividad agrícola y uso de fertilizantes, incremento de la población e incorporación de aguas residuales municipales y/o el efecto de plantas de piscicultura, donde la alimentación de los peces introduce nutrientes, todo lo cual favorece el desarrollo de florecimientos.

No existen en Chile, normativas referentes a la calidad de aguas en relación a toxinas de cianobacterias. La OMS estableció como valor de referencia 1 ug/L como máximo aceptable para el consumo diario de microcistinaL-R.

Deberían considerarse como medidas precautorias, muestreos periódicos para detectar las cianobacterias y ante la presencia de un florecimiento, debería reemplazarse el agua de abastecimiento, hasta comprobar la no toxicidad de la misma, o la reducción de toxinas a valores aceptables. Además, deberían controlarse las poblaciones de cianobacterias regulando los parámetros que favorecen los florecimientos y la toxicidad. 


\section{REFERENCIAS}

Azevedo,S.M.;Evans,O.;Carmichael,W,R.\& Namikoshi, W.W. (1994). First report of microcystin from a Brazilian isolated of the cyanobacterium Microcystis aeruginosa. Appl. Phycol. 6:261-265

Bauld, J. (1981). Ocurrence of benthic microbial mats in saline lakes. Hydrobiología. 81:87-111

Bothe, H.(1982). Nitrogen fixation. In: Carr, N.G \& Whitton,B.A (Ed). The Biology of cyanobacteria University of California Press, Berkeley.

Brock, T.D.(1976). Halophilic blue-green algae. Arch. Microbiol.107:109-111

Bryant, D.A. (1986). The cyanobacterial photosynthetic apparatus comparison to those of higher plants and photosynthetic bacteria. In:Plant,T. \& Li,W.K.W.(Ed). Photosynthetic picoplankton. Call.Bull.Fish. Aquat.Sci. 214:71-120

Cabrera S.; Montecino,V.;Vila,I.; Bahamondes,N.; Barends,I.; Rodríguez, R.; Ruiz, R.; Soto, D.(1997). Características Limnológicas del Embalse Rapel, Chile Central . Medio Ambiente y Represas, Tomo I, pp. 40-60

Campos,H.;Steffen,W.;Román,C.;Zúñiga.L.;Aguero,G. (1983). Limnological studies in Lake Villarrica, Morphometric, , physical, chemitry planktonical factors and primary prodictivity. Arch.Hidrobiol.Suppl. 65:371-406

Campos, H.;Steffen, W.; Aguero, G.; Parra, O.;Zúñiga, L. (1992 a). Limnology of Lake Ranco. Chile. Limnologica 22:337353

Campos, H.; Steffen,W.; Parra,O.; Domínguez, P.; Aguero.G. (1987a). Estudios Limnológicos en el Lago Caburgua (Chile). Gayana.Bot. 44:61-84

Campos, H.;Steffen,W.; Aguero,G.; Parra,O.; Zúñiga,L. (1992 b). Limnological Studies of Lake Rupanco (Chile) Morphometry, Physics, Chemistry, Plankton and Primary productivity.Arch. Hidrobiol. /Suppl.90 (Monographische Bertrage) $1: 85-113$

Campos, H.; Arenas,J.;Steffen,W.;Aguero,G.;Parra,O.; Zúñiga, L.(1990). Limnological study of Lake Todos Los Santos (Chile). Morphometry, Physics, Chemistry, Plankton and Primary productivity. Arch. Hidrobiol. 117:453-484

Campos, H.; Arenas,J.;Steffen,W.;Aguero,G.;Parra,O.; Zúñiga, L. (1987 b). Limnology of Lake Riñihue. Limnol (Berlín) 18:339-357

Campos, H.; Arenas,J.;Steffen,W.;Aguero,G.;Parra,O.; Zúñiga, L. (1988).Limnological study of Lake LLanquihue. Morphometry, Physics, Chemistry, Plankton and Primary productivity. Arch. Hidrobiol. / Suppl. 81, 1:37-67

Campos,V.; Cantarero,S.;Urrutia,H.;Heinze,R.;Wirsing, B.; Neumann, U.;Weckesser,J. (1999). Microcystin in Cyanobacterial Blooms in a Chilean Lake. System Appl.Microbiol. 22:169-173

Campos. V.; Prado, B.; Lizama, C.; Péndola, L.; Robledano,
M. (1990). Prokariotes of Saline environment in the Atacama Salar. Proceedings Second Bienal Water Quality Symposium. 69:275 $-279$

Carmichael, W.W. \& Falconer, I.R.(1993). Diseases related to freshwater blue-green algal toxins, and control measures. In: Falconer, I.R(eds).Algal Toxins in Seafood and Drinking Water. Academic Press, London. pp.197-209

Carmichael, W.W.(1994). The toxins of cyanobacteria. Scient. Amer. 270:64-72

Castenholz, R. (1982). Motility and taxis. In: N.G Carr \& B.A Whitton (Ed). The Biology of cyanobacteria University of California Press, Berkeley. pp. 413-439

Castenholz, R. \& Utkilen, H. (1984). Physiology of sulfide tolerance in a thermophilic Oscillatoria . Archives of Microbiology 138:299-305

Ciferri .O. (1983). Spirulina, the edible microorganism . Microbiol. Rev. 47:551-578

Codd. G.A.; Lawton, W.P. \& Beattie, L.A. (1989). Cyanobacterial toxins in European waters. Ocurrence, properties, problems and requirement. In: Wheller. D.Richardson, M.J. Bridges (eds). The future for water Quality in Europe.Pergamon Press, Oxford. pp.211-220

Codd, G. (1995). Cyanobacterial toxins: Occurrence, properties and biological significance. Wat Sci. Rech. 32:149-156

Cohen - Bazire \& Bryant, D. (1982). Phycobilisomes: composition and structure. In: N.G.Carr and B.A Whitton (Ed) . The Biology of cyanobacteria University of California Press, Berkeley. pp.143 -190 .

Cohen, Y.; Jorgensen,B.B.; Revbech, M.P. \& Poplawski, R.(1986). Adaptation to hydrogen sulfide of oxygenic and anoxygenic photosynthesis among cyanobacteria. Appl. Environ. Microbiol. 51:398-407

Dellarossa, V.; Ugarte, E. \& Parra, O. (1976). Estudio limnológico de la Laguna Chica de San Pedro, La Posada y Lo Mendez. Aspectos cuantitativos del fitoplancton invernal y su relación con algunas características físico químicas del ambiente. Bol.Soc. Biol de Concepción. Tomo L:87-101

Erikson,J.; Toivola,D.D.; Meriluoto, J.; Kurase, H.; Hans,H.; Harrtshorne, D. (1990). Hepatocyte deformation induced by cyanobacterial toxins reflects inhibiton of protein phosphatases. Biochem. Biophys. Res.Comm. 173:1347-1353

Fogg, G.E.; Stewart,W.D.P.; Fay, P. \& Walsby, A.E. (1973). The blue-green algae. Academic Press. London.

Friedmann, E. I. (2001). http://ciencia.nasa.gov/headlines/y2001/ ast26jan_1.htm

Golecki, J. \& Drews, G. (1982). Supramolecular organization and composition of membranes. In: N.G Carr and B.A Whilton (Ed). The Biology of cyanobacteria University of California Press, Berkeley.pp.125-141 
Javor, B.J. \& Castenholz, R.W. (1981). Laminated microbial mats, Laguna Guerrero Negro, México. Geomicrobial. J. 2:237274

Jochimsen, E.; Carmichael, W.W.; Cardo, J.; Cookson,D.W.; Holmes, S.T.; Antunes, B.; Melo-Filho, D.A.; Lyra, D. T.; Spinelli, T.; Baretto,V.; Azevedo, S.M.F.; Jarvis, W.R. (1998). Liver failure and death after exposure to microcystins at a hemodialysis center in Brazil. New England Med. 338:873-878

Muhlhauser, H.A. (1990). Organic Matter Mineralization in the flocculent layer of on intermittent hypertrophic lake. Proceedings Second Biennal Water Quality Symposium

Neumann, U.; Campos, V.; Cantarero, S.; Urrutia, H.; Heinze, R.; Weckesser, J.; Erhard, M. (2000). Co-Ocurrende of Non-toxic (Cyanopeptolin) and Toxic (microcystin) Peptides in a Bloom of Microcysts sp fron a Chilena Lake. System.Appl. Microbiol. 23: 191-197

Paerl, H.W. (1996). A comparison of cyanobacterial bloom dynamics in freshwater, estuarine and marine environment. Phycology 35 (suppl):25-35

Parra, O.; Dellarosa, V. \& Ugarte, E. (1976). Estudio limnologico de la Lguna Chica de San Pedro, La Posada y Lo Mendez. Análisis cualitativo y cuantitativo del plancton invernal. Bol. Soc. Biol. de Concepción. Tomo L:75-86

Pouria, S. A.; Andrade, De.; Barbosa, J.; Cavalcanti, R.L.; Barreto, V.T.S.; Ward, C.; Preiner,W.; Poon, P. (1998). Fatal microcystin intoxication in haemodialysis unit in Caruaru, Brazil. The Lancer 352:21-26

Rapala, J.; Sivonen, K.; Lyra, C. \& Niemela, S. (1997). Variations of Microcystin, Cyanobacterial Hepatotoxins, in Anabaena spp. As a finction of Growth Stimuli. Applied and Environmental Microbiology 2206-2212

Rippka, R.; Waterbury, J.B. \& Stanier, R.Y. (1981). Isolation and purification of cyanobacteria: Some general principles. In: M.P Starr, H.Stolp, H.G. Truper, A. Balws \& H.G. Schlegel (Eds).The prokaryotes. Vol 1. Springer-Verlag, Berlín. pp. 212-220

Rippka, R.; Deruelles, J.; Waterbury, J.B.; Herdman, M.; Stanier, R.Y. (1979). Generic assignments, strain histories and properties of pure cultures of cyanobacteria. J. Gen. Microbiol. 111:1-61

Scarafia, M.; Agnese, E. \& Cabrera, A.M. (1995). Microcystis aeruginosa: behavior and toxic features in San Roque dam (Argentina). Nat. Toxins 3:73-77

Seaburg, K.G.; Parker, B.C.; Wharton, A.R.Jr.; Simmons, G.M. Jr. (1981). Temperature-growth responses of algal isolates from Antarctic oases. J.Phycol.17:353-360
Skulberg, O.; Wood, G. \& Carmichael, W.,W. (1994). Toxic blue-green algal blooms in Europe: A growing problem. Ambio 13: 244-247

Sivonen, K. \& Jones, G. (1999). Cyanobacterial toxins. In: I. Chorus and J.Bartram (Eds), Toxic Cyanobacteria in Water. Guide to Their Public Health Consequences, Monitoting and Management. WHO, E\& FN Spon, London. pp.41-111

Stal .L. (1995). Physiological ecology of cianobacteria in microbial mats and other communities. New Phytol. 131:132

Stanier, R.Y.; Sistrom,W.R.; Hansen,T.A.; Whitton, B.A.; Castenholz, R.W.; Pfening, N.P.; Gorlenko, N.V.; Komdratieva, E.N.; Eimhjellen, K.E.; Whittenbury, R.L.; Trupper, H.G. (1978). Proposal to place the nomenclature of the cyanobacteria (blue-green algae) under the rules of the International Code of Nomenclature of Bacteria. Int .J. Syst. Bacteriol. 28:335 $-336$

Van Liere, L. \& Walsby, A.E. (1982). Interactions of cyanobacteria with light. In: N.G Carr \& B.A Whitton (Eds) . The Biology of cyanobacteria University of California Press, Berkeley. pp.9-45

Vezie, C.; Rapa, J.; Vaitomaa, J.; Seitsonen, J.; Sivonen, K. (2002). Effect of Nitrogen and Phosphorus on Growth of Toxic and Nontoxic Microcystis Strains and on Intracellular Microcystin concentration. Microbial Ecology 43:443-454

Vila, I.; Barends, I. \& Montecino, V. (1987). Abundancia y distribución temporal del fitoplancton en el Embalse Rapel. Chile Central. Revista Chilena de Historia Natural. 60:37-55

Waterbury, J.B.; Watson, S.W.; Valois, F.W. \& Franks, D.G. (1986). Biological and ecological characterization of marine unicellular cyanobacterium Synechococcus. In: T. Plant and W.K. W. Li (Ed), Photosynthetic picoplankton. Can. Bull. Fish. aquat. Sci. 214:71-120

Weckesser, J.; Martin, C. \& Jakobi, C. (1996). Depsipeptides from cyanobacteria: structures and biological activities. J. Syst. Appl. Microbiol 19:133-138

Yoshizawa,S.; Matsushima, R.; Watanabe, R.; Harada, M.F.; Ichihara, K.L.; Carmichael, A.; Fujiki, W.W. (1990). Inhibition of protein phosphatases by microcysin and nodularin associated with hepatotoxicity. Canc. Res. Cli. Oncol. 114:609-614

Zúñiga, L.; Campos, V.; Pinochet,H. \& Prado, B.(1991). A limnological reconaissance of Lake Tebenquiche. Salar de Atacama. Chile. Hidrobiologie. 210:1-24 